\title{
Packaged integrated opto-fluidic solution for harmful fluid analysis
}

T. Allenet, D. Bucci, F. Geoffray, F. Canto, L. Couston, et al.

T. Allenet, D. Bucci, F. Geoffray, F. Canto, L. Couston, E. Jardinier, J.-E. Broquin, "Packaged integrated opto-fluidic solution for harmful fluid analysis," Proc. SPIE 9750, Integrated Optics: Devices, Materials, and Technologies XX, 975011 (15 March 2016); doi: 10.1117/12.2212646

SPIE. Event: SPIE OPTO, 2016, San Francisco, California, United States 


\title{
Packaged integrated opto-fluidic solution for harmful fluid analysis
}

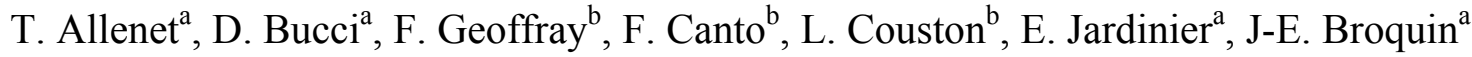 \\ ${ }^{a}$ Univ. Grenoble Alpes, IMEP-LAHC, F-38000 Grenoble, CNRS, IMEP-LAHC, F-38000 Grenoble, \\ France \\ ${ }^{\mathrm{b}}$ CEA Nuclear Energy Division, Radiochemistry \& Processes Department, Marcoule F-30207 \\ Bagnols-sur-Cèze, France
}

\begin{abstract}
Advances in nuclear fuel reprocessing have led to a surging need for novel chemical analysis tools. In this paper, we present a packaged lab-on-chip approach with co-integration of optical and micro-fluidic functions on a glass substrate as a solution. A chip was built and packaged to obtain light/fluid interaction in order for the entire device to make spectral measurements using the photo spectroscopy absorption principle. The interaction between the analyte solution and light takes place at the boundary between a waveguide and a fluid micro-channel thanks to the evanescent part of the waveguide's guided mode that propagates into the fluid. The waveguide was obtained via ion exchange on a glass wafer. The input and the output of the waveguides were pigtailed with standard single mode optical fibers. The micro-scale fluid channel was elaborated with a lithography procedure and hydrofluoric acid wet etching resulting in a $150 \pm 8 \mu \mathrm{m}$ deep channel. The channel was designed with fluidic accesses, in order for the chip to be compatible with commercial fluidic interfaces/chip mounts. This allows for analyte fluid in external capillaries to be pumped into the device through micro-pipes, hence resulting in a fully packaged chip. In order to produce this co-integrated structure, two substrates were bonded. A study of direct glass wafer-to-wafer molecular bonding was carried-out to improve detector sturdiness and durability and put forward a bonding protocol with a bonding surface energy of $\gamma>2.0 \mathrm{~J} \cdot \mathrm{m}^{-2}$. Detector viability was shown by obtaining optical mode measurements and detecting traces of $1.2 \mathrm{M}$ neodymium (Nd) solute in $12 \pm 1 \mu \mathrm{L}$ of $0.01 \mathrm{M}$ and $\mathrm{pH} 2$ nitric acid (HNO3) solvent by obtaining an absorption peak specific to neodymium at $795 \mathrm{~nm}$.
\end{abstract}

Keywords: Integrated optics, microfluidics, optofluidics, chemical analysis, spectral analysis.

\section{INTRODUCTION}

Research in the field of nuclear energy has been moving towards the development of nuclear safety following the accidents of $20^{\text {th }}$ and $21^{\text {st }}$ centuries. Nowadays, efforts are being made to improve the recycling process of nuclear fuel. Research has shown that the separation of radio-nuclides can improve the fuel recycling chain and facilitate conditioning and storage ${ }^{1,2}$. The complex chemical separation methods developed require systematic effluent chemical analysis. Analyses are currently performed inside chemical labs where radioactive samples from the recycling plant are transferred. This complex procedure entails long response times, user and environmental safety issues, as well as high costs. Therefore, the development of the nuclear fuel recycling chain requires new means of analysis with reduced measurement time and effluent volumes ${ }^{3}$ in order to decrease radioactive dosage and therefore reduce risks for workers and the environment as well as global costs. This can be achieved by moving the test-points at the closest to the processline thanks to harsh environment in-situ chemical analysis tools. Because of the advances made in miniaturized glass optical systems, an integrated microfluidic/optical approach is promising given the glass chemical resistance, the microfluidic effluent volume decrease and the many efficient optical detection methods for chemical analysis.

So far, development has been led to introduce more versatile techniques than current industrial in-line methods. A first approach in optical sensors is based on the measurement of phase shift or refractive index differences such as interferometers ${ }^{4}$ and thermal lenses ${ }^{5}$. Although they are quite sensitive, these sensors are wavelength dependent and do not allow spectrometric measurements. Another approach has been the measurement of intensity shifts such as fluorescence or absorption spectroscopy ${ }^{6}$ on a wide spectral range. These techniques are particularly sought out by chemists: they are label-free and allow for broad-band measurements in the NIR-VIS range, which covers many radioactive elements' chemical signature. Several sensor designs based on glass have shown promising results in terms of reproducibility and sensitivity for the analysis ${ }^{7-9}$. However, the actual microfluidic aspect of these designs is very limited if not theoretical: droplets were deposited on sensor surface or straight channels were grinded with a diamond 
blade. Fluids spread into the device from droplets set down on channel/chip edges thanks to the microfluidic capillaryforce induced flow. Although this intrinsic self-induced flow enables measurements with simple fluid droplets and is quite convenient for chip development on a lab bench, the fluid propagation is slow, not adjustable and requires manual handling of the chemicals.

We have chosen to direct our study towards sensor durability and microfluidic function elaboration in order to be able to interface the chips into an extended sensing system with optical and fluid delivery systems. This should allow wellcontrolled transport and pretreatment of sample volumes and dissolutions to come into-play. The addition of optical packaging should also allow deported operation of the chip, which is a first step towards in-line measurements.

In this paper we will start by introducing the device principle of operation as well as the main requirements in terms of both optical and microfluidics functions. Then, the design of the optical waveguide is presented before the realization of the microfluidic chip is detailed. Finally, the first results concerning the optical characterization of the device are shown.

\section{DEVICE PRINCIPLE OF OPERATION}

The desired packaged optical and fluidic integrated device has to provide spectral analysis measurements of small volumes of harsh chemicals in the near infrared and visible range (NIR-VIS). Absorption spectroscopy is an optical means of chemical analysis that provides spectral measurements via the interaction of light and a fluid, with regards to Beer-Lambert's law:

$$
I_{L}=I_{0} \cdot \exp [-\alpha(\lambda) \cdot L]
$$

with $\mathrm{I}_{0}$ the initial intensity, $\mathrm{I}_{\mathrm{L}}$ the transmitted intensity, $\mathrm{L}(\mathrm{cm})$ the interaction length and $\alpha\left(\mathrm{cm}^{-1}\right)$ the absorption coefficient of the sample.

This interaction can be obtained with a co-integrated device by guiding a propagating light wave through an analyte fluid providing there is enough interaction between the two. The co-integration of an optical core adjacent to a microfluidic channel on a chip allows for interaction through the evanescent part of a propagating guided mode ${ }^{10,11}$. In the case of absorption spectroscopy of chemical solutions with evanescent wave interaction, the absorption coefficient becomes $\alpha=\Gamma \cdot \varepsilon \cdot \mathrm{C}$ where $\Gamma$ is the interaction coefficient between the evanescent wave and the fluid, $\varepsilon\left(\mathrm{L} \mathrm{mol}^{-1} \mathrm{~cm}^{-1}\right)$ is the molar attenuation coefficient and $\mathrm{C}\left(\mathrm{mol} \mathrm{L}^{-1}\right)$ is the molar concentration of the chemical element. Absorption spectroscopy therefore results in the study of absorption of parts of the light spectrum through the measurement of the absorbance $\mathrm{A}(\lambda)$ :

$$
A(\lambda)=-\log \frac{I_{L}}{I_{0}}=\frac{1}{\ln 10} \cdot \Gamma \cdot C \cdot L \cdot \varepsilon(\lambda)
$$

Glass is stable, chemically resistant, resilient to radiation and optically transparent in the NIR- VIS range of wavelengths in which the analysis will take place. Furthermore, it allows the fabrication of optical cores with the ion-exchange technology, which makes the use of glass substrates very suitable for absorption spectrometry. The fabrication of integrated microfluidic functions can be achieved by first etching channels on a glass wafer surface and then encapsulating them with another wafer. However, the handling of harsh chemicals prevents the usability of bonding layers, glue or polymer hoods. Direct wafer-to-wafer bonding of glass substrates has hence been developed for this hybrid opto-fluidic device. To be easily used in a glove box environment, the sensor fluidic functions must be interfaced with external capillaries through commercially available fluidic chip-mounts. The proposed device is schematically represented on Figure 1. It consists of one glass wafer with optical functions implemented on it that is bonded to another glass wafer containing the microfluidic functions. Since the refractive index of the fluid is lower than the one of the waveguide's core, when the microfluidic channel is bonded on the top of the waveguide, light remains confined in it and interacts evanescently with the probed chemical element. 

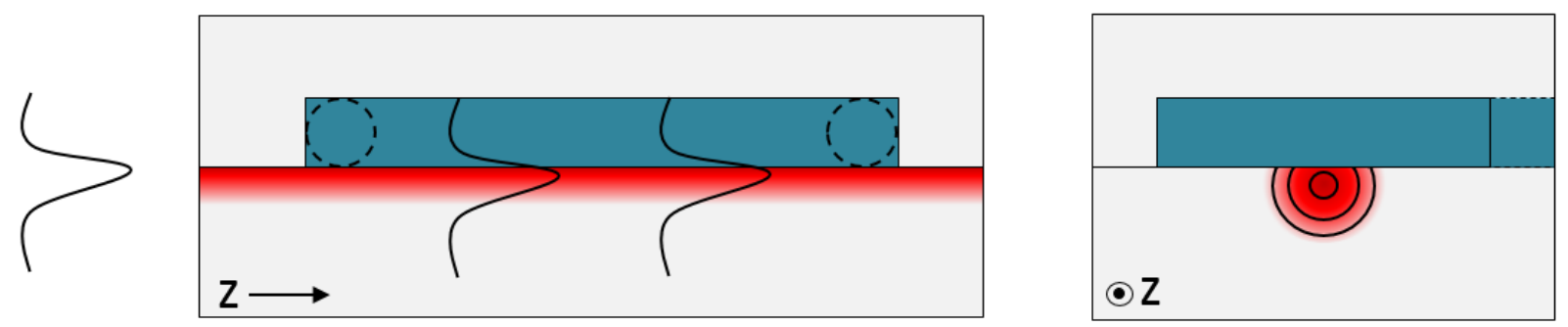

Figure 1. Cross-sectional views of a co-integrated device with two bonded substrates in gray, fluid capillaries and channel in blue, optical core in red and propagating field in black.

Many of the chemical elements that this device aims to detect are actinides with chemical absorption bands in the NIRVIS range. Within the frame of this proof of concept study, neodymium (Nd) has been chosen as chemical model for element detection. Indeed, $\mathrm{Nd}$ is a lanthanide which is chemically similar to important radioactive chemicals $(\mathrm{U}, \mathrm{Np})$ while being non-toxic itself. $\mathrm{Nd}$ therefore allows a first measurement approach with limited handling precautions. The absorption spectrum of $\mathrm{Nd}$ is displayed on Figure 2 where three absorption peaks within the $0.7 \mu \mathrm{m}-0.9 \mu \mathrm{m}$ range can be seen. Therefore, this wavelength range has been selected as the operation range of the device and the optical waveguides must be designed to confine light over it.

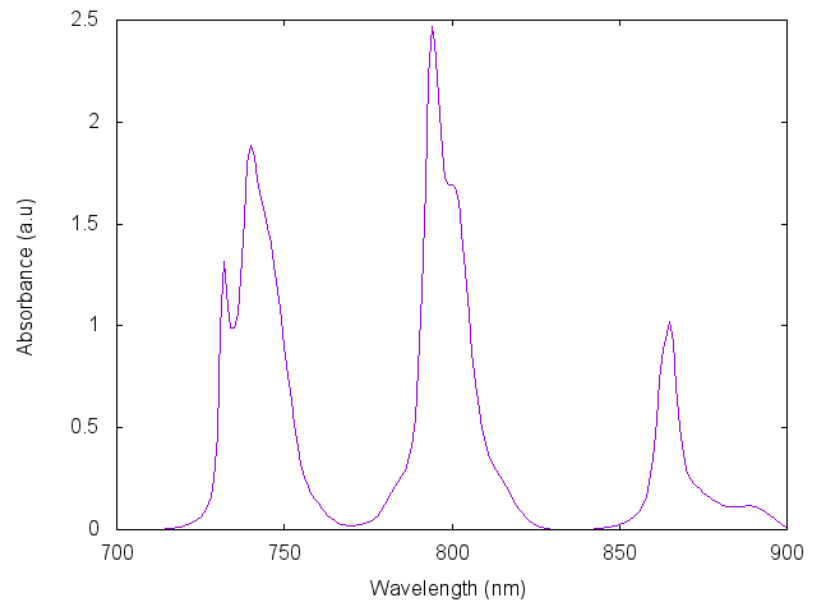

Figure 2. Measurement of $\mathrm{Nd}$ absorption spectrum with a $[0.4 \mathrm{M}] \mathrm{Nd}+[0.01 \mathrm{M}] \mathrm{HNO}_{3}$ solution and the commercial Shimadzu UV 1800 spectrophotometer, which requires $2 \mathrm{~mL}$ of fluid.

\section{DESIGN OF THE OPTICAL WAVEGUIDE}

Ion exchange on glass allows fabricating integrated waveguides by locally increasing the refractive index of a wafer ${ }^{12,13}$. The technique consists in heating a glass wafer in order to provide enough energy to break bonds between modifying ions and the silicate glass matrix without affecting the glass structure. By putting the glass into contact with an external source of ions with the same polarity as the modifying one, a site-to-site ion exchange is carried-out as shown schematically in Figure 3. Substituting an ion by another one changes the refractive index locally because of the change of ion size and polarizability. Therefore, by carefully selecting substituting ion-pairs, exchange times and designing diffusion apertures, one can accurately control the variation of refractive index with or without induced structural constraints, in order to create cores of waveguides. 


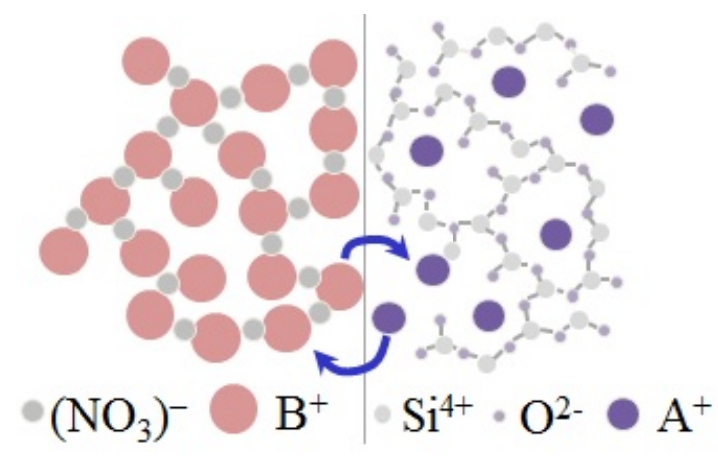

Figure 3. Ion exchange principle with dopant ions $\left(\mathrm{B}^{+}\right)$in the external ion source substituting sites with modifying ions $\left(\mathrm{A}^{+}\right)$in the silicate glass matrix.

The realization of waveguides on glass by ion exchange has been extensively reported ${ }^{12,13}$. It starts by the deposition of a masking layer (in our case $\mathrm{Al}_{2} \mathrm{O}_{3}$ ), which is patterned by photolithography in order to define the waveguides' diffusion apertures. Then the glass wafer is plunged into a molten salt containing dopant ions in order to create the waveguide core by diffusion of the exchanged ions. After removal of the masking layer, the obtained core can be buried within the wafer thanks to another ion exchange assisted by an electric field or left at the wafer surface. Since, a surface interaction between the guided mode and the fluid is required, the first solution has been selected. The key parameters for the design of an ion-exchanged waveguide are: the temperature, which impacts the coefficients of diffusion of the two ions; the diffusion aperture, which contributes to the waveguide width and finally, the exchange duration, which determines both the depth and width of the waveguide's core. The design of the waveguide has been performed thanks to a procedure described in detail by J.-E. Broquin ${ }^{12}$ : first the doping ion concentration distribution is computed thanks to a Finite Difference solving of the diffusion equations, then the waveguide refractive index distribution is deduced and it is injected in a scalar mode solver (here Optimode by Optiwave ${ }^{\mathrm{TM}}$ ) in order to determine the number of guided modes as well as their respective characteristics. Given the harsh nature of the detection medium, the use of borosilicate glass is of interest for it is particularly sturdy and non-brittle to the nuclearized environment. The commercially available Schott BF33 0 borosilicate glass contains $4 \%$ of sodium $\left(\mathrm{Na}^{+}\right)$ions which allows the creation of optical cores with a silver/sodium ion exchange $\left(\mathrm{Ag}^{+} / \mathrm{Na}^{+}\right)$, it has therefore been selected for this study. Figure 4 shows the waveguide that has been designed on this glass. For a diffusion aperture of $7 \mu \mathrm{m}$, an exchange duration of $10 \mathrm{~min}$ and a temperature set to $353^{\circ} \mathrm{C}$, light confinement was obtained from $\lambda=700 \mathrm{~nm}$ to $\lambda=1 \mu \mathrm{m}$.
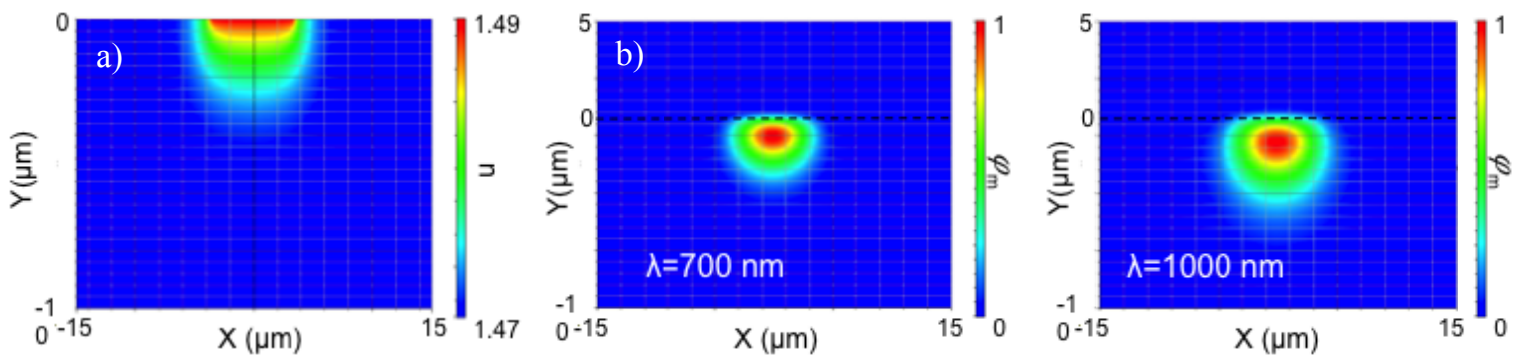

Figure 4: a) Refractive index distribution of the designed waveguide, b) guided mode field distribution at $\lambda=700 \mathrm{~nm}, \mathrm{~b}$ ) guided mode field distribution at $\lambda=1 \mu \mathrm{m}$.

With these simulations an interaction coefficient with $\mathrm{HNO}_{3}$ acting as the $\mathrm{Nd}$ solvent has been computed to be $0.31 \%$ at $\lambda=795 \mathrm{~nm}$ where the absorption of $\mathrm{Nd}$ is maximum. In order to verify whether this rather low interaction coefficient is high enough to allow a measurement, a simple test has been carried-out. Droplets of $[0.8 \mathrm{M}] \mathrm{Nd}+[0.01 \mathrm{M}] \mathrm{HNO}_{3}$ have been deposited on the waveguide over its entire length $(3.8 \mathrm{~cm})$ and an absorption measurement has been performed using a fiber coupled halogen lamp as a source and an USB 2000 OCEAN OPTICS spectrometer. As can be seen on Figure 5, the absorption spectrum of $\mathrm{Nd}$ can clearly be identified, which qualifies this evanescent field approach and allows the cointegration with microfluidic functions. 


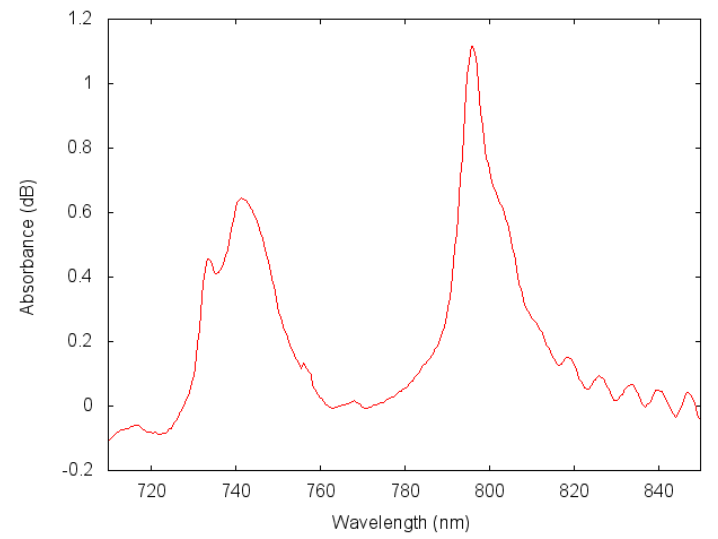

Figure 5. Evanescent wave absorbance spectrum of $[0.8 \mathrm{M}] \mathrm{Nd}+[0.01 \mathrm{M}] \mathrm{HNO}_{3}$ droplets deposited on a $4 \mathrm{~cm}$ long and $3 \mu \mathrm{m}$ wide waveguide measures with a halogen lamp light source.

\section{MICROFLUIDIC CHIP DEVELOPMENT}

\subsection{Design constraints}

Because the microfluidic functions have to be compatible with standard mounts and the bonding of the two wafers must be molecular, the fabrication of the device entails the following constraints:

- Wafer surfaces must be clean and exhibit low roughness to be molecularly bonded. The fluidic channel etching procedure should not therefore impact the wafer surface that must remain intact.

- The bonding between the two wafers must be sturdy and resist to dicing, polishing procedures and fluid infiltrations.

- Channel depth must be greater than the penetration depth of the evanescent wave in the fluid.

- Analyte volume must be small and allow interfacing with exterior capillaries.

In the following paragraphs we will investigate fabrication techniques, design the device's fluidic functions and establish fabrication protocol by treating the constraints listed ahead through quantified objectives.

\subsection{Wafer bonding investigation for glass}

Molecular bonding is a low-temperature $\left(\mathrm{T}<150^{\circ} \mathrm{C}\right)$ direct wafer-to-wafer bonding process, which allows bonding flat silicate wafers without additives, simply by surface reorganization. It is widely used for silicon and silicon oxide wafers. Bonding strength depends on the attraction and adhesion of two hydrophilic surfaces and its quality is enhanced with the number of hydroxyl $(\mathrm{OH})$ groups at the surface. The quality of the process therefore lies on the cleanness of the wafer surfaces and the environment as well as surface activation procedure used to increase the number of $\mathrm{OH}$ groups. The bonding itself is essentially based on three mechanisms: spontaneous adhesion, slow fracture effect and consolidation by annealing $^{14-17}$.

The Double Cantilever Beam (DCB) test ${ }^{15}$ is a widespread method used to quantify substrate bonding strength which provides surface energy measurements. The surface energy measurement is a derivative of bonding strength. We will therefore use surface energy measurements of a bonded structure as a quantification of its bond quality. A surface energy threshold of $\gamma=1.0 \mathrm{~J}^{-2}{ }^{-2}$ is needed for the bond to be resistant to dicing and polishing procedures ${ }^{14}$.

In order to carry out a measurement, a thin blade is inserted between the bonded wafers, which results in a crack opening as sketched on Figure 6. 


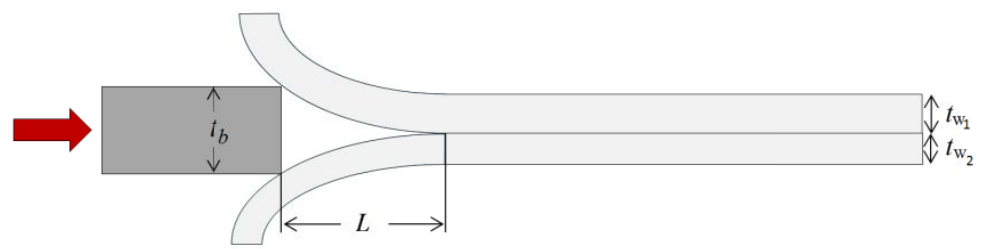

Figure 6. Principle of the DCB test crack-opening length measurement for bond strength characterization

By measuring the crack opening length, we can assess the surface energy of the wafers according to the equation:

$$
\gamma=\frac{3 \mathrm{t}_{\mathrm{b}}^{2} \mathrm{E}_{1} \mathrm{t}_{\mathrm{w} 1}^{3} \mathrm{E}_{2} \mathrm{t}_{\mathrm{w} 2}^{3}}{16 \mathrm{~L}^{4}\left(\mathrm{E}_{1} \mathrm{t}_{\mathrm{w} 1}^{3}+\mathrm{E}_{2} \mathrm{t}_{\mathrm{w} 2}^{3}\right)}
$$

with $\gamma$ the surface energy, $L$ the crack-opening length, $t_{b}$ the blade thickness and $t_{w i}$ and $E_{i}$ wafer thickness and Young's modulus respectively. Given that we bond two identical substrates, equation (3) becomes:

$$
\gamma=\frac{3 \mathrm{t}_{\mathrm{b}}^{2} \mathrm{t}_{\mathrm{w}}^{3} \mathrm{Et}_{\mathrm{w}}^{3}}{32 \mathrm{~L}^{4}}
$$

In order to study surface energy of bonded BF33을 substrates, a measurement system was set.
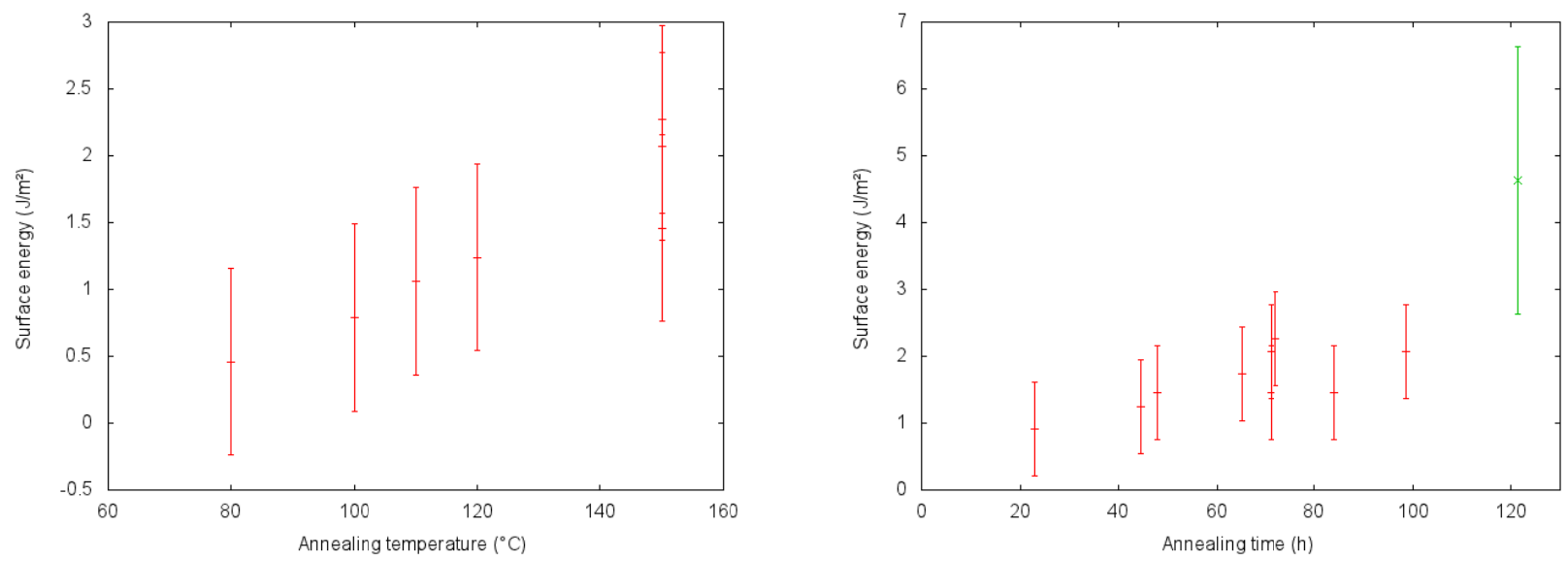

Figure 7. DCB test surface energy measurements as a function of temperature and time. $\gamma(\mathrm{T})$ measurements (left) were carried out with $150^{\circ} \mathrm{C}$ annealing and $\gamma(\mathrm{t})$ measurements (right) were carried out with $72 \mathrm{~h}$ annealing.

Figure 7 shows the impact of the annealing temperature and time on the surface energy. It can be noticed that surface energy increases with annealing time and almost linearly with annealing temperature. Samples annealed for $120 \mathrm{~h}$ at $150^{\circ} \mathrm{C}$ and for $72 \mathrm{~h}$ at $200^{\circ} \mathrm{C}$ could not be separated with the blade during the test without being completely destroyed. This suggests that their surface adhesion was greater than the actual wafer mechanical strength. However, in order to apply bonding to optical integrated devices, we must find a compromise between annealing time and temperature, and optical core diffusion. Given the measured surface energies and the aimed threshold, annealing temperatures and times above $150^{\circ} \mathrm{C}$ and $72 \mathrm{~h}$ respectively will not be considered.

We have obtained substrate bonding with measured surface energy of $\gamma=2.3 \pm 0.4 \mathrm{~J} \mathrm{~m}^{-2}$. With regards to this study, an enhanced molecular bonding procedure ${ }^{17,18}$ was put forward with increased comprehension of experimental parameters. According to our survey, the enhanced protocol should exhibit bonding with $\gamma>2.3 \mathrm{~J} \mathrm{~m}^{-2}$, which is greater than the 1.0 $\mathrm{J} \mathrm{m}^{-2}$ estimated to resist standard mechanical aggression, including chip dicing and polishing. 


\subsection{Micro-channel realization}

The fabrication of microfluidic functions is achieved by etching micro-scale channels. In order to fabricate the proposed device, we have some constraints on the channel depth, which should be sufficiently small in order to decrease effluent volumes while being deep enough to prevent blockade from the analyte fluids, which are initially filtered at $20 \mu \mathrm{m}$. We therefore aim for etching depths of $100 \mu \mathrm{m}$ to steer clear of fluidic function malfunction and to maximize light/fluid interaction while dealing with internal chip volumes smaller than the external capillary ones.

Deep etching technologies include mechanical etching, laser ablation, wet etching with concentrated hydrofluoric acid (HF) and dry etching with plasma enhanced ion bombardment: Inductively Coupled Plasma Reactive Ion Etching (ICPRIE). Mechanical etching techniques include drilling, ultrasonic drilling ${ }^{20}$, electrochemical discharge ${ }^{20}$. Although these methods show high etching rates, they are limited in precision and pattern flexibility. They are also rather aggressive technologies presenting an issue with surface and channel roughness, which are key to the substrate bonding process. Powder blasting ${ }^{21}$ also exhibits high etching rates, but etch precision is limited and very closely linked to etch depth leading to a high level of constraints in etching profile. In addition, powder blasting requires particular instruments and installations. Laser ablation of $100 \mu \mathrm{m}$ deep channels with a precision of $15 \mu \mathrm{m}$ has been demonstrated without use of any controlled environment and little constraint on substrate surface ${ }^{16}$. However, this technology is limited in terms of volume of ablated matter.

Dry etching of glass with ICP has also been investigated. The intrinsic mechanisms of the dry etching process (ionic bombardment) renders directional etching with (quasi-)vertical walls. Anisotropic etching and compatibility with lithography patterning can result in very high precision etching. The limiting factor in the dry etching technology resides in the choice of masking mask layer. Deep channels have been produced by using thick amorphous silicon (a-Si) or Nickel (Ni) layers as well as glass/Si wafer bonded masking layers ${ }^{1622-25}$. However, putting these masking layers into place can be time consuming (substrate bonding) or extremely polluting for the dry etching instruments.

The slightly more traditional chemical wet etching of substrates with concentrated hydrofluoric acid exhibits a fast etching rate. This technology is also compatible with lithography: high precision etching can be obtained, though it is lower than dry etching due to the isotropic behavior which leads to pattern enlargement. Dry and wet etching of glass substrates require a classical clean room environment for masking layer elaboration and lithography patterning.

It is debatable that both laser ablation and the dry etching technology are the most promising for microfabrication in terms of precision. However, they are maturing technologies and require further development. Given the depth and precision standards sought out to elaborate integrated capillary-like channels, the wet etching technology is sufficient in our line of work.

1.

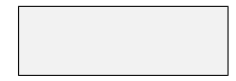

4.

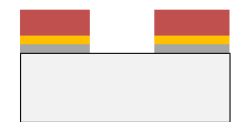

2.
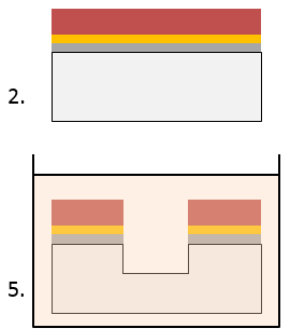

3.

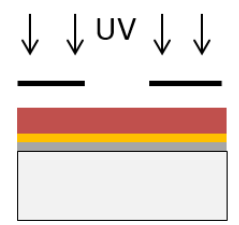

6.

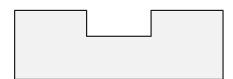

Figure 8. Wet etching process flow for microfluidic function elaboration on glass. 1. Substrate cleaning 2. Sputter deposition of Chrome and Gold layers and elaboration of $10 \mu \mathrm{m}$ SPR220 photoresist. 3. Patterning with lithography 4. Removal of exposed $\mathrm{Cr}$ and $\mathrm{Au}$ layers with chemical etchant 5. HF etching 6. Masking layer removal

Etch depths of $100 \mu \mathrm{m}$ were achieved with an etch rate of $6.3 \pm 0.3 \mu \mathrm{m} \cdot \mathrm{min}^{-1}$ by following the process flow described on Figure 8. It was observed that thick SPR-220 photoresist was chemically inert to HF. A stack of $\mathrm{Cr} / \mathrm{Au}$ layers elaborated by consecutive sputter depositions is necessary for photoresist adhesion and HF etching. This series of metal layers also helps prevent the appearance of defects in the masking layer. Moreover, SPR-220 photoresist is a hydrophobic matter. This has the advantage of helping to prevent the infiltration of etching chemicals into possible photoresist pin-holes. 


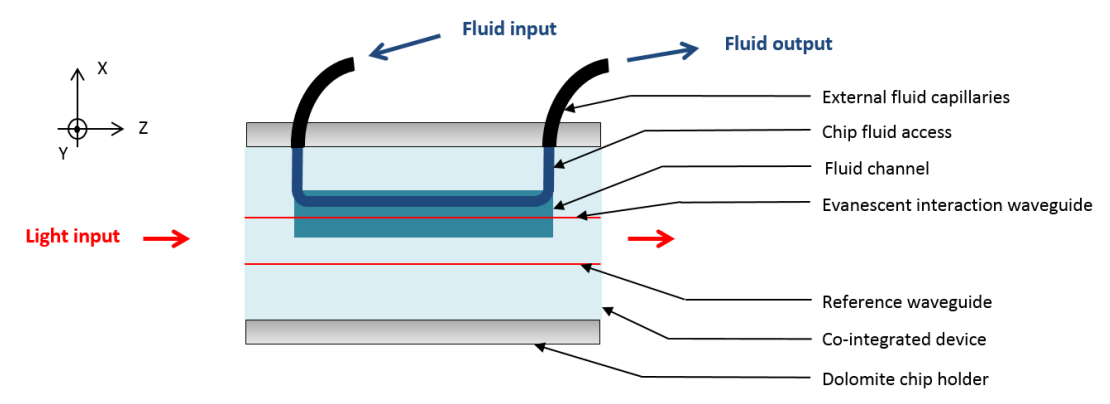

Figure 9. Microfluidic function design for packaging of the chip with a commercial chip holder

A lithography mask was designed to pattern the device's fluidic function in order to place the optical and fluidic inputs/outputs on different sides of the device. The sensing chip has also been designed to fit inside a commercial holder by Dolomite (C), used for microfluidic interfacing (Figure 9). In order to avoid fluid resistance and leakage at the chip external interface and given that external capillaries are $250 \mu \mathrm{m}$ in diameter, the fluid accesses mask opening was designed to be $100 \mu \mathrm{m}$ wide to obtain channels that are approximately $250 \mu \mathrm{m}$ wide and $125 \mu \mathrm{m}$ deep after the HF isotropic etching.

\subsection{Microfluidic qualification}

Having established direct wafer bonding and etching procedures, the microfluidic chip has been realizes. An optical microscope image taken of the fluidic access channels is shown in Figure and shows $250 \mu \mathrm{m}$-wide and $125 \mu \mathrm{m}$-deep channels. The bowl-like channel shape is due to the rounded pattern created by the isotropic wet etching and the flat cover. The fluid channel dimensions for the evanescent field interaction is $10 \times 8 \times 0.125 \mathrm{~mm}^{3}$. Its width is intentionally large in order to cover more than one optical waveguides of the device.

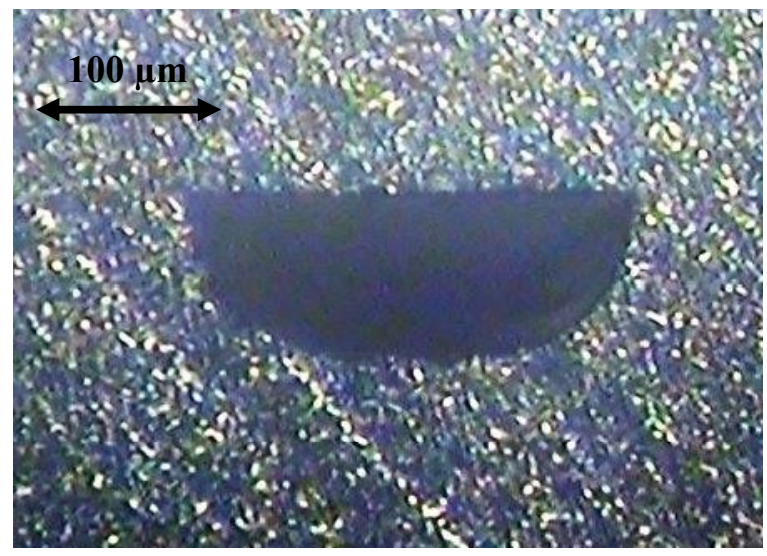

Figure 10. Cross-sectional photograph of a fluidic access channel fabricated with glass etching and bonding procedures.

Using the commercial interface, we have connected the fabricated chip with external capillaries and succeeded in pumping fluid from external recipients in and out of the device simply by applying a vacuum pressure to the fluid output capillary (Figure ). The microfluidic control of the chip has therefore been brought to simple valve control and can be automated, allowing for sample pretreatment. 


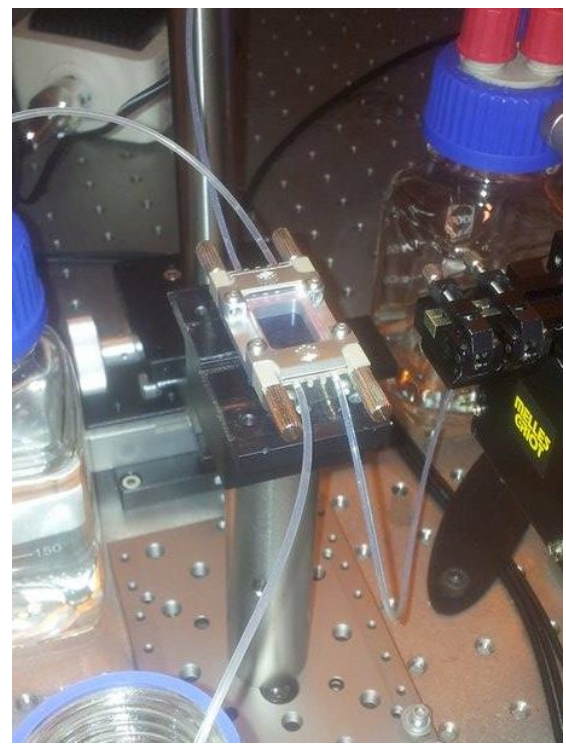

Figure 11. Microfluidic chip positioned in a commercial mount and interfaced with an external fluidic control system.

\section{FIRST RESULTS OF COINTEGRATION AND PERSPECTIVES}

Both fluidic and optic functions having been qualified separately, a complete device has been realized and tested. It has been mounted on the DOLOMITE mount for microfluidic control and its cross-sectional modal response was measured with an infrared camera in order to verify light confinement through the reference and evanescent field waveguides. The device was then implemented in an optical bench system with a LEUKOS supercontinuum light source and an Ocean Optics USB2000+ VIS-NIR digital spectrometer for absorption spectrum measurements.

This first prototype was used to measure $1.2 \mathrm{M} \mathrm{Nd}$ in a $\mathrm{HNO}_{3}$ solution with a $7 \mu \mathrm{m}$-wide waveguide. Analyte fluid was pumped through external capillaries into device fluidic channel by using the Dolomite $\mathbb{C}$ connector.

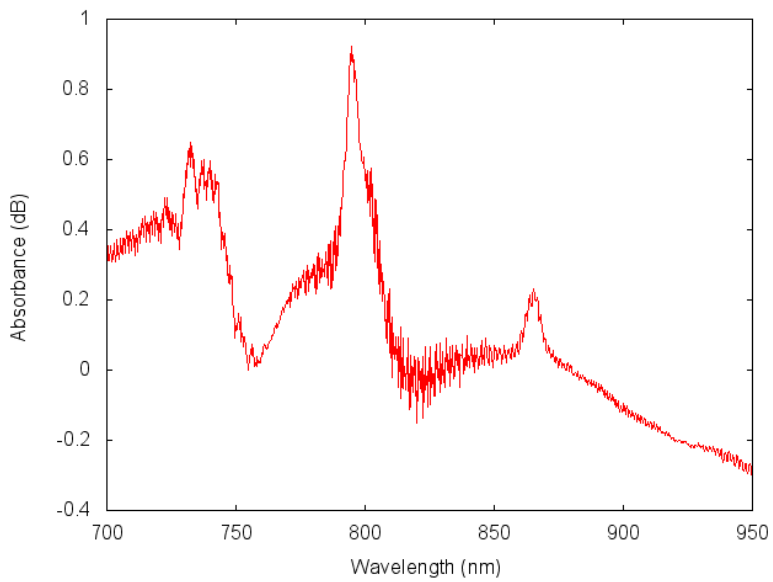

Figure 10. Evanescent wave absorbance spectrum of $[1.2 \mathrm{M}] \mathrm{Nd}+[0.01 \mathrm{M}] \mathrm{HNO}_{3}$ pumped into an integrated microfluidic channel. The evanescent field interaction is $1 \mathrm{~cm}$ long and the waveguide is $7 \mu \mathrm{m}$ wide. Measurements were made with a LEUKOS light source and an Ocean Optics digital spectrometer.

As can be seen on Figure 10, we were able to measure three clear Nd absorption peaks in the 0.7 to $0.9 \mu \mathrm{m}$ wavelength range. This first result is very encouraging though the signal noise suggests that the fluid sample preparation, the fluid/waveguide interaction and the signal normalization should be improved. However, this result is of significant interest for it proves we have succeeded in measuring the same spectrum as the commercial spectrometer in Figure 2 with a fully packaged co-integrated device. 


\section{CONCLUSION}

A glass integrated optic device was designed to allow broadband wavelength transmission for absorption spectroscopy measurements in the NIR-VIS range. A low-temperature direct wafer bonding method has been implemented for glass wafer bonding, and a protocol was put forward to ensure bonding sturdiness. Wet etching of glass substrates has also been investigated in order to create desired microfluidic functions. Finally, the technological advances discussed were tested with the fabrication and preliminary characterization of a co-integrated optical and fluidic device used for chemical analysis in harsh environments. We successfully measured traces of a diluted chemical element with the fabricated glass device proving wafer bonding sturdiness and microfluidic flow control. The spectral range of the analysis is of state-of-the-art character for integrated device and is currently bordered by fiber optic and waveguide cutoff wavelengths. The experiment proves the feasibility of the method and now opens the path to improvement of measurement sensitivity. Improvement of the sensor lies in the increase of the interaction between the light and the fluid. According to Beer-Lambert's law this can be achieved by optimizing interaction length either by increasing device dimensions or by winding the waveguide into spiral optical cores. On the other hand, improving the interaction coefficient through novel designs can also result in increased device sensitivity. On the chemical analysis aspect of this study, the developed system can already be used to investigate pre-treatment of sample volumes, measurement statistical treatment, deported operation and automation.

\section{REFERENCES}

[1] Guillaumont, R., "Radioactive wastes: management by separation-transmutation," Tech. Ing. Genie Nucleaire, BN3663.1-BN3663.16 (2010).

[2] Lecomte, M., Bonin, B., "Traitement-recyclage du combustible nucléaire usé," CEA Saclay; Groupe Moniteur (2008).

[3] Janssens-Maenhout, G., Buyst, J.., Peerani, P., "Reducing the radioactive doses of liquid samples taken from reprocessing plant vessels by volume reduction," Nucl. Eng. Des. 237(8), 880-886 (2007).

[4] Luff, B. J., Wilkinson, J. S., Piehler, J., Hollenbach, U., Ingenhoff, J.., Fabricius, N., "Integrated Optical MachZehnder Biosensor,” J. Light. Technol. 16(4), 583 (1998).

[5] Schimpf, A., Bucci, D., Nannini, M., Magnaldo, A., Couston, L.., Broquin, J.-E., "Photothermal microfluidic sensor based on an integrated Young interferometer made by ion exchange in glass," Sens. Actuators B Chem. 163(1), 29-37 (2012).

[6] Moulin, C., Briand, A., Decambox, P., Fleurot, B., Lacour, J. L., Mauchien, P.., Remy, B., “Techniques d'analyses d'actinides et de radioéléments d'intérêt par spectroscopie laser," Radioprotection 29(04), 517-538 (1994).

[7] Conzen, J.-P., Bürck, J.., Ache, H.-J., "Characterization of a Fiber-Optic Evanescent Wave Absorbance Sensor for Nonpolar Organic Compounds," Appl. Spectrosc. 47(6), 753-763 (1993).

[8] Bernini, R., Campopiano, S., Zeni, L.., Sarro, P. M., "ARROW optical waveguides based sensors," Sens. Actuators B Chem. 100(1-2), 143-146 (2004).

[9] Barrios, C. A., Gylfason, K. B., Sánchez, B., Griol, A., Sohlström, H., Holgado, M.., Casquel, R., "Slotwaveguide biochemical sensor," Opt. Lett. 32(21), 3080 (2007).

[10] Hill, K. O., Watanabe, A.., Chambers, J. G., "Evanescent-Wave Interactions in an Optical Wave-Guiding Structure," Appl. Opt. 11(9), 1952 (1972).

[11] Pandraud, G., Koster, T. M., Gui, C., Dijkstra, M., van den Berg, A.., Lambeck, P. V., "Evanescent wave sensing: new features for detection in small volumes," Sens. Actuators Phys. 85(1-3), 158-162 (2000).

[12] Broquin, J.-E., "Glass integrated optics: state of the art and position toward other technologies," Proc. SPIE $6475,647507-13$ (2007).

[13] Tervonen, A., West, B. R.., Honkanen, S., "Ion-exchanged glass waveguide technology: a review," Opt. Eng. 50(7), 071107-071107-15(2011).

[14] Plößl, A.., Kräuter, G., "Wafer direct bonding: tailoring adhesion between brittle materials," Mater. Sci. Eng. R Rep. 25(1-2), 1-88 (1999).

[15] Maszara, W. P., Goetz, G., Caviglia, A.., McKitterick, J. B., "Bonding of silicon wafers for silicon-on-insulator," J. Appl. Phys. 64(10), 4943-4950 (1988).

[16] Queste, S., Salut, R., Clatot, S., Rauch, J.-Y.., Malek, C. G. K., "Manufacture of microfluidic glass chips by deep plasma etching, femtosecond laser ablation, and anodic bonding," Microsyst. Technol. 16(8-9), 1485-1493 (2010).

[17] Alam, A. U., "Surface Analysis of Materials for Direct Wafer Bonding," thesis (2014). 
[18] Xu, Y., Wang, C., Dong, Y., Li, L., Jang, K., Mawatari, K., Suga, T.., Kitamori, T., "Low-temperature direct bonding of glass nanofluidic chips using a two-step plasma surface activation process," Anal. Bioanal. Chem. 402(3), 1011-1018 (2011).

[19] Xu, Y., Wang, C., Li, L., Matsumoto, N., Jang, K., Dong, Y., Mawatari, K., Suga, T.., Kitamori, T., "Bonding of glass nanofluidic chips at room temperature by a one-step surface activation using an O2/CF4 plasma treatment," Lab. Chip 13(6), 1048 (2013).

[20] Yang, C. T., Ho, S. S.., Yan, B. H., "Micro Hole Machining of Borosilicate Glass through Electrochemical Discharge Machining (ECDM)," Key Eng. Mater. 196, 149-166 (2001).

[21] Schlautmann, S., Wensink, H., Schasfoort, R., Elwenspoek, M.., Berg, A. van den., "Powder-blasting technology as an alternative tool for microfabrication of capillary electrophoresis chips with integrated conductivity sensors," J. Micromechanics Microengineering 11(4), 386 (2001).

[22] Kolari, K., Saarela, V.., Franssila, S., "Deep plasma etching of glass for fluidic devices with different mask materials," J. Micromechanics Microengineering 18(6), 064010 (2008).

[23] Park, J. H., Lee, N.-E., Lee, J., Park, J. S.., Park, H. D., "Deep dry etching of borosilicate glass using SF6 and SF6/Ar inductively coupled plasmas," Microelectron. Eng. 82(2), 119-128 (2005).

[24] Goyal, A., Hood, V.., Tadigadapa, S., "High speed anisotropic etching of Pyrex ${ }^{\circledR}$ for microsystems applications,” J. Non-Cryst. Solids 352(6-7), 657-663 (2006).

[25] Akashi, T.., Yoshimura, Y., "Deep reactive ion etching of borosilicate glass using an anodically bonded silicon wafer as an etching mask," J. Micromechanics Microengineering 16(5), 1051 (2006). 